\title{
Pulsating enophthalmos and choroidal hamartomas: two rare stigmata of neurofibromatosis
}

\author{
PETER J. SAVINO, JOEL S. GLASER, AND MALCOLM N. LUXENBERG \\ From the Bascom Palmer Eye Institute, Department of Ophthalmology, University of Miami School of \\ Medicine, and Department of Ophthalmology, Medical College of Georgia
}

SUMMARY A young girl with neurofibromatosis demonstrated two rare stigmata of this diseasepulsating enophthalmos and multiple pigmented choroidal hamartomata. Fundus photographs of the latter are believed to be the first of their kind to be published.

The ocular stigmata of neurofibromatosis, although inconstant and variable, should be familiar to ophthalmologists and other specialists likely to have to diagnose and treat the congenital hamartomatoses. Detailed reviews of the 'neurophakomatoses' are available and need not be elaborated here (Walsh and Hoyt, 1969; Vinken and Bruyn, 1972). We have recently seen a patient with neurofibromatosis who demonstrated two exceedingly rare stigmata of the

This investigation was supported in part by Public Health Service Training Grant No. EY-00028 from the National Eye Institute, National Institutes of Health, Bethesda, Md. 20014

Dr Peter Savino is supported in part by a fellowship from the Heed Ophthalmic Foundation, Inc, Chicago, Illinois

Address for reprints: J. S. Glaser, MD, PO Box 520875, Biscayne Annex, Miami, Florida 33152, USA disease-pulsating enophthalmos and choroidal hamartomata. The illustrations of the latter are, to the best of our knowledge, the first published photographs of these fundus lesions.

\section{Case report}

A 12-year-old right-handed White girl, examined in December 1970, was found to have neurofibromatosis. The right upper eyelid was diffusely thickened without a discrete mass, and the margin showed an S-shaped deformity (Fig. 1). Pulse-synchronous movement of the right globe was observed and exophthalmometry revealed $2-3 \mathrm{~mm}$ of right enophthalmos. The position of the globe could not be altered by head-hanging or Valsalva manoeuvre.

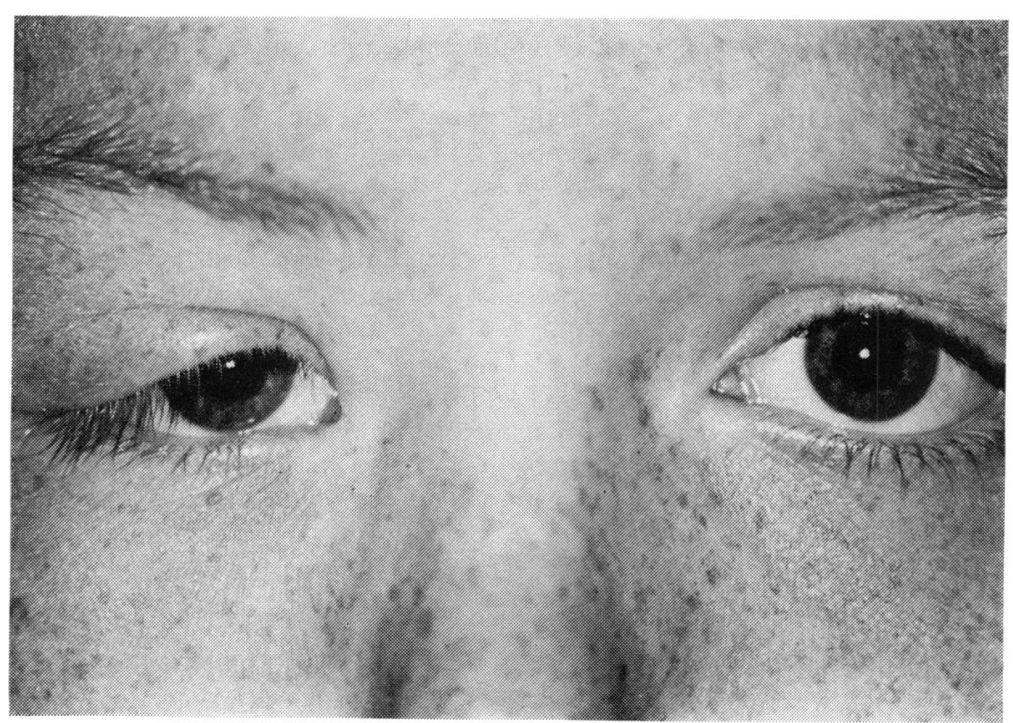

483
Fig. 1 Note $S$-shaped deformity of right upper lid and enophthalmos of right globe 


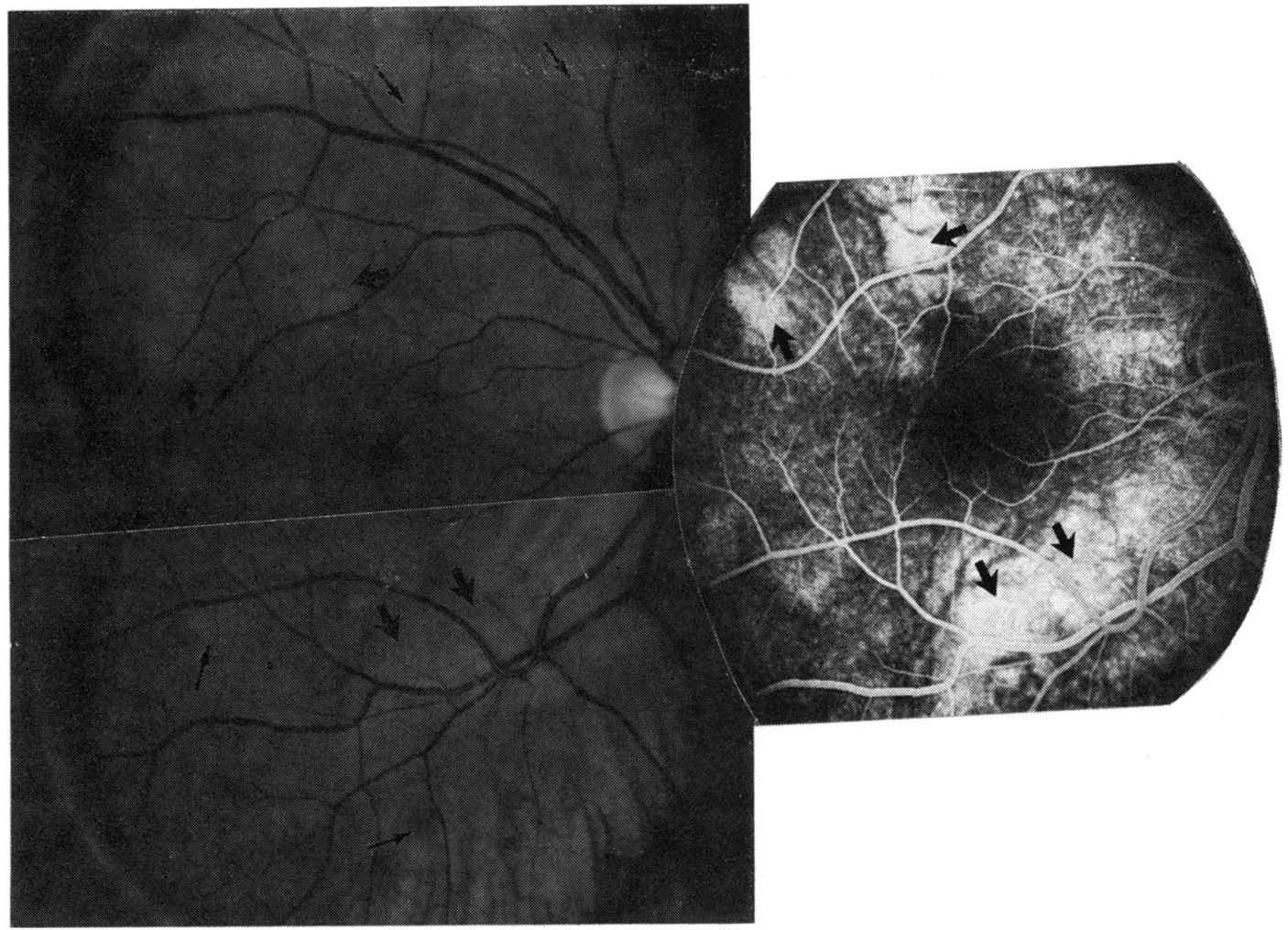

Fig. 2. Fundus colour photographs show small, discrete hyperpigmented choroidal lesions (small arrows) associated with depigmentation of retinal pigment epithelium (large arrows). Black-and-white photograph of arteriovenous phase of fluorescein shows defect of retinal pigment epithelium filter over 'hyperfluorescent' choroidal lesions (arrows). Note that inferior lesion is more extensive than expected from colour photograph

Best corrected vision was right $20 / 20$, left $20 / 15$ (RE: $-4.50+2.00 \times 80^{\circ}$; LE: $-3.50+1.25 \times 90^{\circ}$ ). Ocular motility, colour vision, pupillary responses and Goldmann perimetry were all normal. Slit-lamp examination showed two nodular iris lesions thought to be compatible with fibrous hamartomata.

Ophthalmoscopy showed multiple small, pigmented, circumscribed lesions along with larger hypopigmented areas (Fig. 2). Fluorescein angiography showed a pattern consistent with early choroidal filling defects and larger areas of hyperfluorescence during the arteriovenous phase. These findings were interpreted as areas of absent retinal pigment epithelium overlying variably pigmented hamartomata of the choroid, although histological confirmation was not possible.

Numerous large and small café-au-lait spots were scattered over the patient's body, and a large, raised violaceous fibroma molluscum was seen in the area of the left flank and abdomen. Neurological examination was normal.
Radiological examination of the skull (Fig. 3), including orbital tomography, showed enlargement of the sella with erection of the dorsum plus pronounced deformity of the greater and lesser wings of the sphenoid. The right optic canal and orbital fissure were grossly deformed and the right orbit expanded. These findings were thought to be consistent with sphenoidal dysplasia, an osseous malformation seen in neurofibromatosis.

Ocular, physical, and radiological re-examination in August 1973 showed no changes. Vision was at the same level and the right pulsating enophthalmos persisted. B-scan ultrasonography showed a defect of the right orbit, with no posterolateral wall echo and a suggestion of a temporally located smoothwalled mass.

\section{Discussion}

Neurofibromatosis is a heredofamilial disorder with multivariegated ocular and systemic stigmata. 

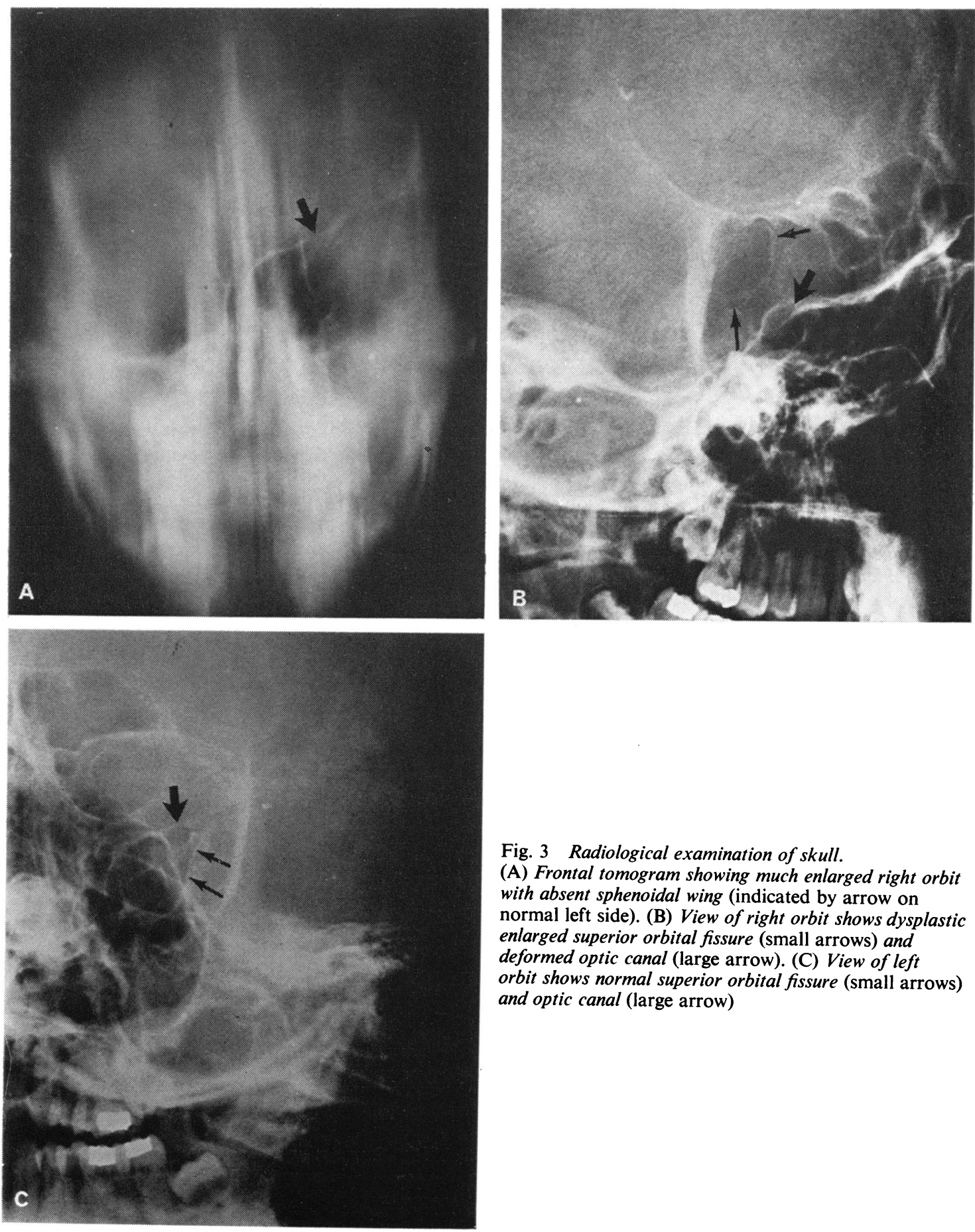

Fig. 3 Radiological examination of skull.

(A) Frontal tomogram showing much enlarged right orbit with absent sphenoidal wing (indicated by arrow on normal left side). (B) View of right orbit shows dysplastic enlarged superior orbital fissure (small arrows) and deformed optic canal (large arrow). (C) View of left orbit shows normal superior orbital fissure (small arrows) and optic canal (large arrow) 


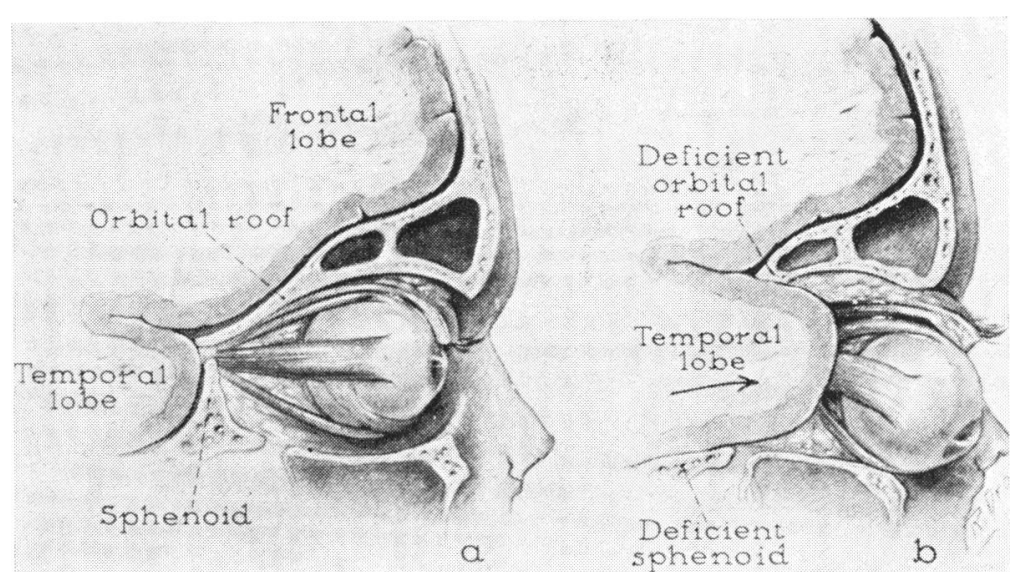

Fig. 4 Diagrammatic representation of mechanism of pulsating exophthalmos in sphenoidal dysplasia (Bruwer, A. J., and Kierland, R. R. (1955))
Exophthalmos, when present, is usually associated with a mass lesion in the orbit or a buphthalmic globe due to congenital glaucoma (Table 1). Exophthalmos accompanied by pulsating protrusion of the eye but without bruit indicates intracranial pulse waves transmitted to the orbital contents via a major congenital defect in the posterior wall of the orbita sphenoidal-orbital encephalocele (Fig. 4). Otherwise, pulsating exophthalmos is occasionally observed with few other lesions (Table 2).

Unilateral enophthalmos, on the other hand, is usually seen after orbital trauma as an early or late complication of orbital floor fracture. Other causes of unilateral enophthalmos must be considered quite rare (Table 3). The pulsating enophthalmos in our case was associated with a radiologically demonstrable sphenoidal dysplasia in a patient with other diagnostic stigmata of neurofibromatosis.

A review of the literature reveals only six potentially similar cases. Petres (1962) reported a case entitled 'Unilateral pulsating exophthalmos and neurofibromatosis'. In fact, the non-pulsating globe was exophthalmic by $4 \mathrm{~mm}$, but 4.50 dioptres more myopic than the pulsating 'enophthalmic' globe. This patient underwent ipsilateral ligation of the carotid and jugular vessels with no relief of symptoms. Orbital $x$-rays were said to show enlargement of the optic foramen as the only radiological abnormality.

In his review of the orbital bony changes in neurofibromatosis Burrows (1963) reported the case of a 48-year-old woman (case 10) with a pulsating enophthalmos and a large ipsilateral defect of the sphenoid bone. Vision and ocular movements are described as 'good' without any further clinical details. Lenshoek et al. (1968) reported the case of a 15-year-old girl with pulsating enophthalmos accompanied by an audible murmur. An exophthalmic phase would appear during periods of emotion.
Enlargement of the ipsilateral orbit with destruction of both sphenoid wings was demonstrable radiologically.

Nikiforov (1966) described the case of a 28-yearold man who during a physical exertion experienced the sudden onset of severe left-sided head pain, diplopia on right gaze, and rapidly progressing left pulsating exophthalmos, followed by loss of consciousness and a seizure. A 'blowing noise', which disappeared on ipsilateral carotid compression, was heard over the left supraorbital region. The deep tendon reflexes were increased on the right side

\section{Table 1 Causes of exophthalmos in neurofibromatosis}

\author{
(1) Orbital tumour \\ (a) Neurilemoma \\ (b) Plexiform neuroma \\ (c) Meningioma \\ (2) Optic nerve glioma \\ (3) Nerve sheath meningioma \\ (4) Sphenoidal-orbital encephalocele \\ (5) Buphthalmos (congenital glaucoma)
}

Table 2 Causes of pulsating exophthalmos

(1) Carotid-cavernous fistula

(2) Sphenoidal-orbital encephalocele (dysplastic, traumatic or surgical)

(3) Orbital venous varix or arteriovenous malformation

(4) Vascular tumours (hemangioma)

\section{Table 3 Causes of enophthalmos}

(1) Orbital floor fracture

(2) Fat atrophy (traumatic)

(3) Facial hemiatrophy (scleroderma, en coupe de sabre)

(4) Sclerosing orbital tumour

(5) Microphthalmos or phthisis bulbi

(6) Orbital fibrosis 
and there was a right positive Babinski sign. Left carotid arteriography showed an inferiorly displaced middle cerebral artery, a left orbital bony defect, but no aneurysm'. The left eye became $12 \mathrm{~mm}$ enophthalmic and later stabilised at $3 \mathrm{~mm}$ enophthalmos. The author speculates that the patient originally had had a longstanding left pulsating enophthalmos associated with neurofibromatosis and that the acute episode was due to herniation of the meninges and temporal lobe into the orbital defect. We believe this case was most likely one of carotid-cavernous fistula with late enophthalmos.

Dabezies and Walsh (1961) recorded the case of a 27-year-old man with pulsatile enophthalmos who, 10 years previously, had sustained left frontotemporal trauma of sufficient force to render him unconscious for three days. Orbital $x$-ray examination revealed bony defects of the sphenoidal wings and the orbital portion of the frontal bone, which was partially absent. The authors suggested that the enophthalmos was due to post-traumatic atrophy of orbital fat.

Finally, Vinken and Bruyn (1972) cite two cases of pulsating enophthalmos. The first (Kelly, 1949) concerns a patient with neurofibromatosis and a large parasellar mass in whom the presence of pulsating enophthalmos is mentioned cursorily with no further details. The second case is that of a patient reported by Franceschetti and Streiff (1938). However, review of the original article shows this to be a case of pulsating exophthalmos (esoftalmo con pulsazione) and not enophthalmos.

It would therefore seem that primary pulsating enophthalmos uncomplicated by trauma or other precipitating events is extremely rare.

In the present case the classical dysplastic defects in the sphenoid bone are sufficient to account for the pulsations, although the enophthalmic position of the globe cannot easily be explained. The radiographic appearance of this dysplasia has been described by Binet et al. (1969) in a study of seven patients thought to have neoplasms or chronic subdural haematomas. It is important to note that, in addition to hypoplasia of the greater sphenoid wing, a raised lesser wing, sellar deformities, and enlarged middle cranial fossa, in all patients the ipsilateral orbit was enlarged. In five patients with optic foramina views the optic canal was enlarged or 'destroyed', but only one patient had visual loss and was found to have an optic nerve glioma. Although the other patients were not surgically explored it was concluded that the abnormal optic foramina were due to the congenital sphenoidal dysplasia.

In our case B-scan ultrasonography showed an abnormality of the retro-orbital fat pad, which

\section{Table 4 Fundus findings in neurofibromatosis}

(1) Optic atrophy associated with optic nerve/chiasm glioma

(2) Gliomatous raising of optic disc

(3) Optic atrophy associated with hydrocephalus

(4) Glaucomatous cupping due to congenital buphthalmos

(5) Choroidal melanoma

(6) Choroidal naevi

(7) Multiple pigmented choroidal hamartomata

(8) ? True retinal glioma

(9) ? Increased incidence medullated nerve fibres

appeared less dense and smaller than in the normal orbit. This finding may account for the enophthalmos. Regarding the fundoscopic findings, choroidal lesions in neurofibromatosis are very rare, although it is said that melanoma occur more often in patients with neurofibromatosis (Table 4) (Font and Ferry, 1972).

Nordmann and Brini (1970) suggest that the association of neurofibromatosis and pigmented tumours reflects a disturbance in the development of neural crest cells derived from neuroectoderm, which in turn give rise to Schwann cells, meningoblasts, and melanocytes of the skin and uveahence the increased incidence of schwannomas, meningiomas, and melanomas in patients with neurofibromatosis. These authors further point out that although nodules and benign melanomata of the anterior segment of the eye are very common in neurofibromatosis such lesions in the posterior pole are exceptional and 'almost certainly not related to the disease'. Included in this publication was a fundus photograph of a large choroidal naevus.

Commenting on such choroidal lesions, Wolter et al. (1962) state that when they do occur they are associated with a typical clinical picture of congenital unilateral glaucoma leading to buphthalmos and blindness. Microscopically the posterior choroid is diffusely thickened, containing many ganglion cells, nerve-fibre bundles, and lamellated 'ovoid bodies' made up of elongated hyperplastic Schwann cells. Wolter (1965) subsequently concluded that nerve fibres actually branch between the onion-like layers of these Schwann cells, thus resembling sensory nerve end-organs.

Although pathological confirmation was impossible, we believe that from the clinical appearance and fluorescein pattern of the choroidal lesions in our case they represented hamartomatous stigmata of neurofibromatosis. We do not know of any previously published fundus photographs.

\section{References}

Binet, E. F., Kieffer, S. A., Martin, S. H., and Peterson, H. O. (1969). Radiology, 93, 829.

Bruwer, A. J., and Kierland, R. R. (1955). Archives of Ophthalmology, 53, 2. 
Burrows, E. H. (1963). British Journal of Radiology, 36, 549.

Dabezies, O. H., and Walsh, F. B. (1961). Transactions of the American Academy of Ophthalmology and Otology, 65, 885.

Font, R. L., and Ferry, A. P. (1972). International Ophthalmology Clinics, 12, 1.

Franceschetti, A., and Streiff, E. B. (1938). Atti della Società Oftalmologica Italiana XXXIV Congress, 34, 365.

Kelly, R. E. (1949). Proceedings of the Royal Society of Medicine, 42, 67.

Lenshoek, C. H., van Manen, J., Notermans, S. L. H., and Penning, L. (1968). Neurochirurgie, 14, 986.
Nikiforov, B. M. (1966). Vestnik Oftalmologii, 79, 77.

Nordmann, J., and Brini, A. (1970). British Journal of Ophthalmology, 54, 641.

Petres, J. (1962). Klinische Monatsblätter für Augenheilkunde, $140,356$.

Vinken, P. J., and Bruyn, G. W. (1972). Handbook of Clinical Neurology, vol 14. North-Holland: Amsterdam.

Walsh, F. B., and Hoyt, W. F. (1969). Clinical NeuroOphthalmology, vol. 3. Williams \& Wilkins: Baltimore.

Wolter, J. R., Gonzales-Sirit, R., and Mankin, W. J. (1962). American Journal of Ophthalmology, 54, 217.

Wolter, J. R. (1965). Archives of Ophthalmology, 73, 696. 